\section{A elaboração de estatísticas de mortalidade segundo causas múltiplas}

\section{Mortality statistics according to multiple causes of death}

\section{Ruy Laurenti}

Centro Brasileiro de Classificação de Doenças

Departamento de Epidemiologia

Faculdade de Saúde Pública

Universidade de São Paulo

Av. Dr. Arnaldo, 715

01246-904 São Paulo, SP - Brasil

E-mail:cbcd@fsp.usp.br

\section{Cássia Maria Buchalla}

Centro Brasileiro de Classificação de Doenças

Departamento de Epidemiologia

Faculdade de Saúde Pública

Universidade de são Paulo

\section{Resumo}

Uma das possibilidades permitidas pelo avanço tecnológico é o estudo da mortalidade por causas múltiplas em contraposição à estatística feita por uma única causa, a chamada causa básica de morte. $\mathrm{O}$ conhecimento das várias doenças que contribuem para uma morte permite que seja avaliada a importância das causas que normalmente não estariam privilegiadas nas estatísticas porque são doenças não caracterizadas como causa básica. Um exemplo é a mortalidade por infarto do miocárdio em pessoas com diabetes mellitus. Este último, neste caso e em outros semelhantes, poderá não ser considerado nas estatísticas, enquanto o infarto o será. Desta forma, no exemplo citado, analisando apenas a causa básica, perde-se a informação sobre a magnitude do diabetes e sua relação com as complicações que levam à morte. A idéia da elaboração de estatísticas de mortalidade segundo causas múltiplas não é nova. No entanto não é realizada de forma sistemática, ainda que vários estudos mostrem suas vantagens. Entre essas vantagens estão a possibilidade de descobrir novas associações de doenças; conhecer informações sobre a natureza das lesões em casos de morte por causas externas (acidentes e violências), entre outras. A existência de computadores e de programas específicos para a elaboração das estatísticas de mortalidade, atualmente em uso, deve permitir que essa idéia seja colocada em prática e que, a partir das estatísticas de mortalidade por causas múltiplas, possibilite melhores e mais específicas ações de saúde.

Palavras-chave: Estatísticas de mortalidade. Causas múltiplas de morte. 
Abstract

The introduction of new technology has made it possible to study multiple causes of death as opposed to mortality statistics based only on the underlying cause of death. The knowledge of all diseases that contribute to one death allows the assessment of the importance of causes that frequently do not appear in mortality statistics, because of those diseases that are not characterized as the underlying cause. An example is the death by myocardial infarction of a person that also has diabetes mellitus. The idea of producing mortality statistics according to multiple causes is not new. However, it is not a routine, although several studies have demonstrated its advantages. Among the advantages is the possibility of finding new associations of diseases and to know the nature of the injury according to the type of accident or violence. The utilization of computers and specific programs for producing mortality statistics enables multiple-cause statistical calculations, a practice that should be stimulated.

Keywords: Mortality statistics. Multiple causes of death.

\section{Apresentação}

A multicausalidade das doenças é aceita sem restrições, mesmo nos casos onde o agente etiológico é bem conhecido, como nos casos de doenças infecciosas. Para cada doença ou agravo à saúde é possível identificar fatores causais relacionados à biologia humana, ao estilo de vida e ao ambiente; pode-se até, em certos casos, identificar fatores ligados à atenção médica.

No caso de mortes, desde John Graunt, em 1662, tem sido atribuído a cada óbito somente uma "causa". Entretanto, o próprio Graunt, o primeiro a produzir dados de mortalidade segundo causas, em sua clássica publicação "Natural and Political Observations in a Following Index and Made Upon the Bills of Mortality", fez comentários a respeito de "morrer devido a uma doença e morrer com uma doença”, segundo descreve Greenwood ${ }^{1}$.

A idéia de que a morte não é devida a uma só "causa" mas a um "grupo de causas” é, também, amplamente aceita.

É necessário esclarecer o que usual e tradicionalmente se chama de "causa". Em mortalidade esta tem o significado de "doença", "síndrome", "processo patológico" e até mesmo "diagnóstico". Desta maneira, o que vem sendo chamado, há décadas, de "causas múltiplas de morte" ou, simplesmente, "múltiplas causas", seria mais apropriado ou correto chamar de "múltiplas doenças" ou "associações de doenças (ou diagnósticos, ou patologias) responsáveis pela morte".

Assim, conservando o termo "causa" de morte, no sentido estrito, a análise de causas múltiplas de morte, deveria incluir todos os fatores - biológicos, ambientais, estilo de vida - responsáveis pela morte e não somente as doenças. Isto é: a morte e sua multicausalidade.

Entretanto, como tradicionalmente consagrado, a análise, o estudo ou mesmo uma simples tabulação das várias doenças e suas complicações presentes no momento da morte informadas no atestado de óbi- 
to, constituem o que se denomina "causas múltiplas de morte".

No entanto, apesar do Atestado de Óbito permitir que várias doenças sejam colocadas, indicando uma seqüência entre elas, apenas uma é selecionada para as estatísticas, a denominada causa básica da morte.

\section{Importância de se obter estatísticas de mortalidade segundo causas múltiplas}

A importância de se obter tabulações segundo causas múltiplas, e não somente pela causa básica de morte, reside no fato de que raramente uma morte é devida a uma só doença. Assim, se deseja ter um quadro da situação de saúde na população, usando indicadores de mortalidade, o ideal é utilizar todas as doenças e complicações destas, presentes no momento da morte.

Esse fato foi reconhecido pela própria Organização Mundial da Saúde (OMS) logo após sua criação. Assim, na sexta revisão da Classificação Internacional de Doenças (CID), de 1948, a primeira a ser proposta pela OMS, foi apresentado um modelo de tabela para causas múltiplas e sugerida sua utilização ${ }^{2}$. A Oitava Revisão da CID, de 1965, refere-se às causas múltiplas de morte, da seguinte maneira: "Foram apresentados na Conferência trabalhos sobre a necessidade e os problemas da realização de análise de causas múltiplas em grande escala e a possibilidade de fazer estudos mais modestos. A Conferência, embora assinalando a importância da causa básica de morte para a apresentação de séries históricas, reconheceu a necessidade de complementar as estatísticas de causa única com a computação total das afecções notificadas no atestado médico da causa de morte e, ainda mais importante, com a identificação das síndromes dos estados patológicos que, associados, causaram a morte ${ }^{3}$.

Em um trabalho de 1956, "The Enigme of Cause of Death" ${ }^{4}$, o autor discute a questão comentando ser falso admitir que a morte seja o resultado de uma única causa e que o próprio formato do atestado de óbi- to mostra isso; além do mais, continua o autor, a seleção de uma só causa para a finalidade de tabulação tornou-se um problema nem sempre fácil de reconhecer. De fato, a seleção de uma causa, quando várias são informadas, leva muitas vezes à necessidade de usar regras e instruções - muitas vezes arbitrárias - "para grande aflição dos médicos, epidemiologistas e estaticistas".

A transição epidemiológica levou a uma mudança do padrão de mortalidade, com declínio grande das doenças infecciosas e uma considerável diminuição da mortalidade nos primeiros anos de vida. Passou a predominar a mortalidade de adultos e de idosos, prevalecendo, nesta, as doenças não infecciosas: cardiovasculares, neoplasias, diabetes e outras.

$\mathrm{O}$ aumento da vida média faz com que os adultos e idosos passem a "acumular" doenças; assim, a análise da mortalidade por causas múltiplas seria muito apropriada nesses casos. É de se ressaltar, entretanto, que isso também é verdadeiro no caso das doenças infecciosas, mesmo em crianças menores de 5 anos, o que foi comentado, já em 1924 por Dublin e Van Buren 5 . Estes autores fizeram referências às doenças infecciosas comentando que a análise da mortalidade por causas múltiplas seria um instrumental de grande auxílio para os profissionais da Saúde Pública devido à riqueza de informações que contém. Isso também ficou sobejamente mostrado por Puffer e Serrano ${ }^{6}$, na investigação internacional sobre mortalidade de crianças menores de 5 anos.

Em nosso meio, o primeiro autor a discutir e publicar sobre "causas múltiplas" foi Laurenti ${ }^{7}$ que mostrou vários tipos de associações de doenças responsáveis pela morte, entre outros aspectos. É da sua publicação, em 1973, a tabela que se segue mostrando, como exemplo, o diabetes mellitus como causa básica, como causa associada da morte e o total (causas múltiplas) em uma amostra de óbitos no município de São Paulo. 
Tabela - Diabetes mellitus como causa básica e não básica de morte e suas associações com outras doenças em amostras de 1827 atestados de óbito ocorridos em hospitais do Município de São Paulo, 1972.

Table - Diabetes mellitus as underlying cause and not as underlying cause of death and its association with other diseases. Sample of 1827 deaths certificates, both sexes and all ages. Deaths in hospitals, São Paulo municipality, 1972.

\begin{tabular}{lccc}
\hline Diabetes & $\begin{array}{c}\text { C. básica (\%) } \\
(\mathrm{n}=36)\end{array}$ & $\begin{array}{c}\text { Não C. básica (\%) } \\
(\mathrm{n}=41)\end{array}$ & $\begin{array}{c}\text { Total (\%) } \\
(\mathrm{n}=77)\end{array}$ \\
\hline D. Infeccioções & 19,4 & 19,5 & 19,6 \\
Neop. Malignas & - & 4,9 & 2,6 \\
D. Hipertensiva & 22,2 & 43,9 & 33,7 \\
D. Isquêmica Coração & 27,7 & 34,1 & 31,1 \\
D. Cerebrovasculares & 36,1 & 48,8 & 42,8 \\
Outras Doenças Sistema Geniturinário & 19,4 & 26,8 & 23,7 \\
\hline
\end{tabular}

Fonte/Source: Laurenti ${ }^{7}, 1973$.

Os dados apresentados mostraram a importância de uma análise por múltiplas causas. O diabetes, doença associada a uma série de outras patologias, apareceu não como causa básica, em $53,0 \%$ dos casos. Ainda que os eventos causadores da morte, e foram computados como a causa básica, fossem importantes, é de fundamental interesse em epidemiologia conhecer quantos deles foram conseqüência de diabetes.

Entre outros tipos de associações, o mesmo estudo ${ }^{7}$ mostrou que, quando uma doença hipertensiva era mencionada no atestado de óbito (em qualquer posição), havia uma associação com diabetes em 13,1 \% dos casos, com doença isquêmica do coração $(29,3 \%)$, doença cerebrovascular $(66,1 \%)$ e doenças arteriais $(34,8 \%)$.

Outro estudo ${ }^{8}$ que utilizou atestados de óbitos de adultos de 15 a 74 anos de idade, no município de São Paulo, em um período de 12 meses (1974/75), mostrou que a hipertensão arterial foi selecionada como causa básica da morte em 2,7\% dos casos. A análise por causas múltiplas mostrou que a freqüência da doença era de 30,0\% dos casos, o que permite uma melhor análise por variáveis de interesse.

Um dos melhores exemplos sobre o uso da metodologia de análise da mortalidade por causas múltiplas e da riqueza de informações que ela provê, quer para uso em epidemiologia, quer para planejamento e administração em saúde, foi o estudo internacional coordenado pela Organização Panamericana de Saúde e denominado "Investigação Inter-americana de Mortalidade na Infância" ${ }^{6}$. Nas "Highlights and Recomendations" deste estudo está, entre outras colocações, o seguinte: "Somente por meio do estudo de múltiplas causas foi possível medir a magnitude de problemas de saúde em menores de 1 ano e em crianças de 1 a 4 anos. Análises das causas associadas de morte, em adição às causas básicas, foram necessárias para descobrir importantes relações, tais como a ação sinérgica de doenças infecciosas e deficiência nutricional, e os efeitos das complicações da gravidez e do parto sobre os produtos da gestação”.

Respondendo à pergunta "Quais são as aplicações dos dados sobre causas múltiplas”, Kochanek e Rosenberg ${ }^{9}$ expõem o que se segue:

a) descobrir possíveis associações de doenças que não são conhecidas clinicamente;

b) oferecer informações sobre a natureza das lesões (para causas externas);

c) examinar a variabilidade das práticas quanto ao preenchimento dos atestados de óbito;

d) observações sobre maiores especificidades do que normalmente é disponí- 
vel apenas com a causa básica. Exemplo: mortes por AIDS;

e) conhecer a freqüência de importantes condições que contribuem para a morte e que muitas vezes não são selecionadas como causa básica. Exemplos: doença de Alzheimer, pneumonia, diabetes e outras;

f) estabelecer a complexidade de condições mórbidas que medem os riscos de mortalidade;

g) observar certos perfís de ligação que podem ser comuns. Exemplo: diabetes com processos renais e infarto do miocárdio;

h) usar dados de causas múltiplas em análises multivariadas, visando identificar novos problemas médicos, como exemplo: a AIDS foi identificada a partir de perfis de síndromes de doenças infecciosas que eram individualmente raras, mas começaram a ser identificadas como ocorrendo juntas e eventualmente identificando um novo processo de base ou subjacente;

i) estimar vários tipos de modelos de riscos competitivos.

\section{Definições em causas múltiplas}

Para se obter uniformidade internacional na produção das estatísticas de mortalidade por uma só causa foi definido o que é essa causa (causa básica de morte) e como ela deve ser declarada pelo médico.

Para que a elaboração de estatísticas de mortalidade por causas múltiplas sejam comparáveis é preciso, também, que obedeçam às mesmas definições. Existem propostas de definições que, entretanto, ainda não foram incorporadas na CID. Dessas propostas ${ }^{10,11}$ são apresentadas as definições abaixo:

Causas Intervenientes: São todas as "complicações" ou "conseqüências" da causa básica, ou seja, todas as doenças mencionadas e que são precipitadas pela causa básica. Estas são declaradas nas linhas da Parte I do atestado de óbito, acima da causa básica.
A importância de se conhecer quais as doenças conseqüentes à causa básica reside na possibilidade de prevenção. Nem sempre a causa básica da morte é uma doença ou evento fácil de ser prevenido, nesse caso a prevenção pode se feita através de alguma causa associada. Como exemplo pode-se citar o acidente de motocicleta (causa básica) levando a fratura de crânio e ao coma (causas intervenientes). É mais fácil prevenir a fratura de crânio, com o uso de um capacete metálico, do que prevenir $o$ acidente propriamente dito.

Causas Condicionantes: São aquelas que realmente iniciam a cadeia de eventos que levam a morte (são as verdadeiras causas básicas!), porém, pelas regras internacionais de codificação da causa básica, elas não são codificadas como tal. $\mathrm{O}$ exemplo mais conhecido é o da hipertensão arterial ( a verdadeira causa básica) que pode levar a um acidente vascular cerebral e ao coma (causas intervenientes); nesse caso, pela aplicação das regras, dá-se preferência ao acidente vascular cerebral como causa básica da morte.

Ainda que não sejam as causas selecionadas para as estatísticas, é importante que sejam conhecidas, pois sobre elas é que se deve fazer a prevenção primária da morte.

Causas Contribuintes: São aquelas condições (doenças ou complicações) que não fazem parte da cadeia de eventos que, a partir da causa básica, levam à morte. No atestado de óbito (quando bem declarado pelo médico) são as condições informadas na Parte II.

Causas Associadas: São todas as condições informadas que não constituem nem causa básica, nem interveniente, condicionante ou contributória.

\section{Guias, normas e regras para codificar causas múltiplas}

Além das definições é preciso que existam guias, normas ou regras para que as causas múltiplas sejam codificadas.

Da mesma maneira que há orientações, algumas delas bem arbitrárias, para codifi- 
car a causa básica, o mesmo deveria existir para a codificação das outras causas informadas no atestado de óbito. Entretanto, muitas vezes a codificação é feita não se levando em conta a possibilidade de erros ou má interpretação. Os exemplos de atestados de óbito, abaixo, explicam melhor o que pode ocorrer:

Exemplo 1:

I a) Choque hipovolêmico

R57.1

b) Hemorragia gástrica

K92.2

c) Cirrose hepática

K74.6

d) Alcoolismo crônico

F10.2

Pelo Princípio Geral, a causa básica é o Alcoolismo Crônico (F10.2) que, entretanto, por disposição da CID-10, no caso Regra $\mathrm{C}$, se associa com cirrose hepática (K74.6) e leva ao código K70.3: cirrose hepática alcoólica.

Havendo essa associação, o código K70.3 incorpora a cirrose hepática mais o alcoolismo e este, para a produção de estatísticas de causa múltipla, não precisaria ser codificado. Se isso ocorrer o alcoolismo estará sendo contado duas vezes (K70.3 e F10.2). Exemplo 2:

I a) Broncopneumonia

b) Coma

c) Hemorragia meníngea

I60.8

d) Arteriosclerose cerebral

I67.2

Pelo Princípio Geral, a arteriosclerose cerebral I67.2 é a causa básica informada. Entretanto, por disposição da CID-10 (Regra C, associação), quando, em presença de Hemorragia cerebral (I60.8), esta é que deve ser a codificada.

É de se destacar, porém, que a arteriosclerose cerebral (agora causa condicionante) deve ser codificada, pois há interesse, quer clínico quer epidemiológico, em conhecer essa associação.

Exemplo 3:

I a) Fibrilação ventricular

b) Infarto agudo do miocárdio

I21.9

c) Angina pectoris

d) Arteriosclerose coronária

II Infarto antigo do miocárdio

$\mathrm{I} 25.2$
Nesse caso existem quatro manifestações de uma mesma doença (I21.9, I20.9, I25.1 e I25.2); se todas forem codificadas, isto levaria a uma super enumeração da doença isquêmica do coração. De fato, suponha-se que existam 80 casos como este e, se todos os diagnósticos fossem codificados, haveria 320 casos de doença isquêmica do coração, o que não está correto.

Apenas esses três exemplos servem para mostrar, de maneira clara, que a elaboração de estatísticas de mortalidade por causas múltiplas não consiste simplesmente em codificar todos os diagnósticos informados. São necessárias regras, normas e orientações, como as existentes para a seleção e codificação da causa básica. Os "Centros Colaboradores da OMS Para a Classificação de Doenças", entre os quais o "Centro Brasileiro", vêm trabalhando ativamente nesse sentido.

\section{A produção de estatísticas de mortalidade por causas múltiplas}

Embora há muito tempo venham sendo feitos estudos nessa área, esses quase que se limitam a estudos acadêmicos desenvolvidos por especialistas em estatísticas de mortalidade. Nunca houve e até hoje, praticamente, não existe produção rotineira de dados de causas múltiplas. Isso somente se tornou possível com a introdução e aplicação de automação.

Kochanek e Rosenberg ${ }^{9}$ comentam a ocorrência de uma mudança na produção de estatísticas de mortalidade nacionais devido a intensa aplicação de automação, sendo "o principal elemento desta mudança a saída da codificação somente da causa básica, para a codificação de todas as condições informadas no atestado de óbito e a introdução de um sistema automatizado para determinar a causa básica entre todas as condições codificadas. Esse novo sistema foi denominado ACME, de "Automated Classification of Medical Entities”.

Esse sistema foi implantado nos Estados Unidos na década de 80 e, no Brasil, no estado de São Paulo, em 1983. Isso possibili- 
tou a produção de estatísticas por causas múltiplas, porém, ainda sujeita a imperfeições como as que foram mostradas nos três exemplos acima, visto não haver, em pleno funcionamento, regras ou normas. É de se destacar que, ainda que os bancos de dados contenham todos os múltiplos códigos, não se produzem estatísticas por causas múltiplas de forma rotineira.

Atualmente estão sendo desenvolvidos "softwares" usados rotineiramente para selecionar a causa básica da morte e produzir tabulações de todas as causas incluídas nos atestados de óbito. O "Centro Brasileiro de Classificação de Doenças”, em parceria com o DATASUS, produziu um "software" chamado SCB (Seleção da Causa Básica) que possibilita selecionar a causa básica. $\mathrm{O}$ SCB, ainda em aperfeiçoamento, está sendo usado no país, e pode permitir a produção de estatísticas de causas múltiplas, uma vez estabelecidas certas normas e orientações. O "National Center for Health Statistics” (NCHS), nos Estados Unidos, criou o "International Collaborative Effort on Automating Mortality Statistics" que vem trabalhando intensamente nesse assunto $^{12}$; o "Centro Brasileiro de Classificação de Doenças” vem participando deste trabalho colaborativo.

Um aspecto importante na produção de estatísticas de mortalidade, quer seja por causas múltiplas como por causa básica, é o preenchimento de maneira correta do atestado de óbito pelos médicos. No caso de causas múltiplas é preciso que o médico declare todas as causas, partindo da básica e informando corretamente suas complicações até a causa terminal (causas intercorrentes). Deve também informar, sempre que possível, as causas contribuintes na Parte II. Caso isso não ocorra, é discutível o valor das estatísticas de causas múltiplas.

Considerando que o uso do SCB tem sido cada vez maior, o "Centro Brasileiro de Classificação de Doenças” está aumentando suas atividades educativas junto aos médicos, visando o melhor preenchimento do atestado de óbito. Essas atividades são fundamentais para a melhoria da qualidade estatística de mortalidade e parece estar tendo resultado positivo, em várias áreas do país.

Mesmo sendo possível, atualmente, produzir estatísticas por causas múltiplas, o que se espera é que, em um futuro próximo, sua qualidade seja boa. Isso possibilitará o desenvolvimento de estudos epidemiológicos e, principalmente, possibilitará seu uso em planejamento e administração em saúde. É necessário sair da fase de estudos meramente acadêmicos sobre estatísticas de mortalidade por causa múltipla, para um uso prático mais amplo pelos profissionais de saúde pública.

\section{Summary}

Since John Graunt's publication "Natural and Political Observations in a Following Index and Made Upon the Bills of Mortality" in 1662, only one cause has been attributed to each death. Graunt himself made some comments on "dying due to a disease" and "dying with a disease". The idea that a death is not due to a single cause but to a group of causes is widely accepted. Thus, the importance of obtaining tabulations by multiple causes lies on the fact that rarely a death is due to only one disease. To have a picture of the health status of a population by using mortality indicators, the best would be to tabulate all the diseases and complications presented at the moment of the death. Some examples of association of causes are shown in the paper demonstrating the possibility of their use in epidemiology and in health care management. Several papers have already been published in many countries on the multiple causes of death, including a wide range of approaches: concept, method of analysis, its use in epidemiology and health care management. Regarding the application of multiple cause statistics the follow- 
ing is presented: - Uncovering possible disease associations that are not known clinically, - Supplying information on the nature of the injury (for external causes), · Examining the variability in medical certification practices, · Observing more specificity than is normally available in the underlying cause of death, e.g. AIDS, - Observing the frequency of important conditions contributing to death that are not as frequently selected as the underlying cause, e.g. Alzheimer's, diabetes, pneumonia, $\cdot$ Establishing the complexity of morbid conditions that contribute to levels of frailty and to mortality risk assessment, · Observing profiles of conditions that tie together as common processes (diabetes to nephrites to myocardial infarction), · Using multiple cause data in types of multivariate analyses to identify new medical problems or conditions, e.g. AIDS was identified from profiles of infectious disease syndromes that are individually rare but began to be identified as occurring together and eventually identifying a new underlying dis- ease process, - Estimating various types of dependent competing risk models. The production of statistics according to multiple causes as a routine was only possible with the introduction and application of automation. The first program that permitted to produce these statistics was called ACME, for Automated Classification of Medical Entities and it was utilized in the United States. In order to produce multiple cause statistics it is necessary to have very clear definitions of all the causes declared by the physician in the death certificate, and not only the definition of the underlying cause of death. There are proposals for definitions of what would be called: intervening causes; conditioning causes, contributory and associated causes. Guides for coding multiple causes are also needed: how should the causes on death certificates, other than the underlying cause be coded? Three examples of death certificates and the coding proposed of all the causes declared are presented in this paper.

\section{Referências}

1. Greenwood M. Medical statistics from Graunt to Farr. Cambridge: Universitary Press; 1948.

2. Centers for Disease Control and Prevention, World Health Organization. Manual of the International Statistical Classification of Diseases, Injuries, and Causes of Death: 6th revision. Atlanta, Georgia: CDC/WHO; 1948.

3. Organização Pan-Americana da Saúde. Manual da Classificação Estatística Intenacional de Doenças, Lesões e Causas de Óbito. 8 ${ }^{\text {a }}$ revisão. Washington (DC); 1969 (OPS - Publicação Científica, 190).

4. Treolar A E. The enigme of cause of death. J Am Med Assoc 1956; 162:1376-9.

5. Dublin L I, Van Buren GH. Contributory causes of death: their importance and suggestions for their classification. Am J Public Health 1924; 14: 100-5.

6. Puffer R R, Serrano CV. Patterns of mortality in childhood. Washington (DC): Pan American Health Organization; 1973. (PAHO - Scientific Publication, 262).

7. Laurenti R. Causas múltiplas de morte. [Tese de LivreDocência]. São Paulo: Faculdade de Saúde Pública da USP; 1973.
8. Laurenti R. Epidemiologia da hipertensão arterial. In: Chiaverini R. Doença hipertensiva: diagnóstico, etiopatogenia e tratamento. Rio de Janeiro: Livraria Atheneu; 1980. p.65-87.

9. Kochanek KD, Rosenberg HM. Issues, considerations and examples in the uses of multiple cause of death in United States Government statistics. In: WHO Meeting of Heads of Collaborating Centers for the Classification of Diseases; 1995; Canberra, Australia. Washington (DC) : WHO; 1995. (ESS/ICD/C/95.21).

10. World Health Organization. A suggested methodology for multiple causes. In: WHO Meeting of Heads of Collaborating Centers for the Classification of Diseases; 1988; Uppsalla, Sweden. Washington (DC): WHO; 1988. (DES/ICD/C/88.38).

11. Laurenti R. Multiple causes of death: proposals. In: WHO Meeting of Heads of Collaborating Centers for Classification of Diseases. Washington (DC): WHO; 1993.

12. US Department Health and Human Services, National Center of Health Statistics, Center for Disease Control and Prevention. Proceedings of the International Collaborative Effort on Automating Mortality Statistics. Washington (DC); 1999. (DHHS Publication No(PHS) 99-1252). 